\title{
Self-similar Charge Transport in Gapped Graphene
}

Díaz-Guerrero D. S. ${ }^{1 *}$, Gaggero-Sager L. M. ${ }^{1}$, Rodríguez Vargas I. ${ }^{2}$, \& Naumis G.G. ${ }^{3}$ 1.Facultad de Ciencias, Universidad Autónoma del Estado de Morelos. Cuernavaca Morelos, México. 2. Unidad de Física, Universidad Autónoma de Zacatecas. Zacatecas Zacatecas, México. 3. Instituto Física, Depto. de Física-Química,

Universidad Nacional Autónoma de México (UNAM). Apdo. Postal 20-364, 01000, México D.F., México.

A new type of self-similar potential is used to study a multibarrier system made of graphene. Such potential is based on the traditional middle third Cantor set rule combined with a scaling of the barriers height. The resulting transmission coefficient for charge carriers, obtained using the quantum relativistic Dirac equation, shows a surprising self-similar structure. The same potential does not lead to a self-similar transmission when applied to the typical semiconductors described by the non-relativistic Schrödinger equation. The proposed system is one of the few examples in which a self-similar structure produces the same pattern in a physical property. The resulting scaling properties are investigated as a function of three parameters: the height of the main barrier, the total length of the system and the generation number of the potential. These scaling properties are first identified individually and then combined to find general analytic scaling expressions.

*diazd@uaem.mx 
Graphene 1] is considered as one of the most promising new materials. This first truly two-dimensional crystal [2] has impressive physical properties [1, 3]. As a consequence, graphene is not only important from a technological point of view, but also is considered as an inflexion point in quantum physics. For instance, charge carriers in graphene follow an effective quantum relativistic (Dirac) equation instead of the usual Schrödinger one. This leads to new effects [4 6]. Specially, the scattering produced by external potentials or impurities is different from what is observed in ordinary materials. Charge carriers can be transmitted perfectly due to the Klein effect and random sustitutional impurities can produce multifractal states [7, 8]. Thus, a whole new world is open to investigate the possible effects of external potential geometries, which can be imposed to graphene by different means, as for example, using certain type of substrates, strain, electrostatic gates, impurities, electromagnetic fields, etc. [9, 10].

For applications, an important issue is how to engineer substrate-induced bandgap opening in epitaxial graphene [10]. This leads to investigate how different multibarrier geometries affect the properties of graphene. Among these possible geometries, self-similarity has a paramount importance, since scale invariance is a fundamental property of many natural phenomena, as can be corroborated in reports that ranges from the distribution and abundance of species, temporal occurrence of earthquakes, and even in the growth of complex networks and trees [11 14].

From the technological standpoint, self-similarity can also be exploited to produce useful devices [15 17] . Particularly, photoconductive fractal antennas show an efficient multiband emission of terahertz radiation owing to the self-similarity of the fractal structure [16].

Despite that self-similar structures are getting plenty of attention [18], only a handful of experiments corroborate a self-similar behavior in its physical properties [19 21. Likewise, many theoretical works claim that physical properties, such as tranmission or reflection probabilities, display self-similarity. Unfortunately, most of them are just a matter of visual perception, since the scaling properties are never reported. Even in the case of quasicrystals, which are considered as one of the archetypical examples of self-similar structures, it has been elusive to find the scaling properties of the corresponding physical properties 22, 23].

Here we propose a novel self-similar multibarrier structure in graphene in which the main physical property, namely charge transport, presents a surprising self-similar pattern. This self-similar structure does not produce a similar behavior in a usual semiconducting material described by the Schrödinger equation. Thus, two essential ingredients are shown to be into play: a self-similar structure and quantum relativistic mechanics.

To build our multibarrrier structure in one direction (the $x$ axis), we implement a variant of the middle third Cantor case [24] (see supplementary material) using square barriers as indicated in Fig. 1. We begin with a line-segment of length $L t$, we divide it in thirds and put a rectangular barrier of energy $V_{0}$ in the middle third (its width is $L t / 3$ ). We call this the generation one $(G 1)$. The second step consists in taking the remaining line-segments, divide them into thirds and place within scaled copies of the existing barrier. We scale the height and width of the barrier by a scaling factor of $1 / 3$. Additionally the existing barrier is also scaled in its width. Since scaling is a contraction transformation, each scaled copy will have a unique fixed point, see the fixed point theorem in metric spaces 25]. By fixed point we meant an element of the function's domain that is mapped to itself by the function. In this case, this point is a fixed side of the barriers, which will be chosen as the right-hand side. So, every successive iteration (generation) $(G j)$ of the structure is obtained by repeating the second step of the construction for the remaining line-segmentes, using as the "existing barrier" those added in the last iteration, as shown in Fig. 1.

This potential can be described as follows. The $j$-th generation of the potential will consists of $N_{G j}$ barriers; the corresponding barriers (those added in the $j$-th iteration) has a width $W_{G}(j)$ and energy (height) $V_{G} j$. Such barrier is obtained as a scaled replica of barriers from generation $G(j-1)$,

$$
\begin{gathered}
N_{G j}=2^{G j}-1, \\
W_{G j}=\alpha=\frac{L t}{3^{G j}}, \\
V_{G j}=\frac{V_{0}}{3^{G j-1}} .
\end{gathered}
$$

This bidimensional potential does not depend on the perpendicular direction ( $y$ axis), i.e., $V(x, y)=V(x)$ and can be adapted to graphene [1, 26 28] by means of symmetry breaking substrates [29 31], by gated graphene or in other systems, like optical analogies, with a linear spectrum 32 34.

Let us now study the transmission coefficient of charge carriers in this multibarrier system. For graphene, we use the Viana-Gomes et al. formalism [30] to compute the transmission coefficient of electrons in gapped graphene. The Hamiltonian for the Dirac electrons under a potential $V(x)$ is given by

$$
H=v_{F} \sigma \cdot \mathbf{p}+\sigma_{z} V(x),
$$

where $V(x)=m(x) v_{F}^{2}$ if $x \in\left[x_{1}, x_{2}\right]$ and zero in other case. $V(x)$ defines the region where there is a mass term ( $q$-region) and where carriers are massles Dirac fermions ( $k$-region). The first term of the Hamiltonian is just the 

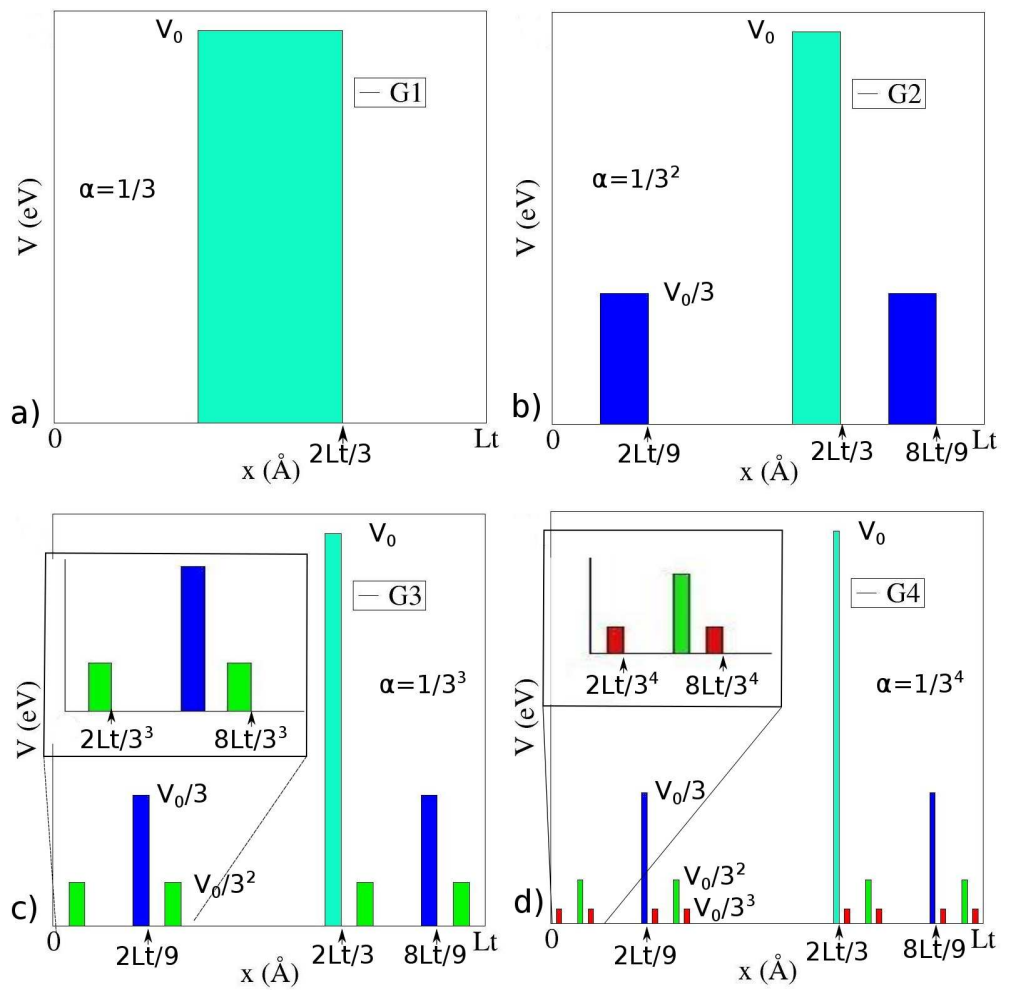

FIG. 1: Potential construction. First four generations (G1,G2,G3,G4) of the multibarrier potential. Each barrier is scaled (we denote the scaling factor by $\alpha$ ) in its width, and so the barrier width is $L t / \alpha$. The barriers added in the last iteration are scaled in its height. For example, the height of the green ones are one third of the height of the blue ones. The zooms in G3 and G4 illustrate how the potential resembles a previous generation after an appropiate rescaling.

Dirac equation used to describe electrons in graphene, where $v_{F}$ is the Fermi velocity, $\sigma$ the set of Pauli matrices and $\mathbf{p}$ the moment. For normal incidence on the $x$ direction the corresponding wavefunctions are 30]

$$
\psi_{k}^{ \pm}(x)=\frac{1}{\sqrt{2}}\left(\begin{array}{c}
1 \\
u_{ \pm}
\end{array}\right) e^{ \pm i k_{x} x}
$$

and

$$
\psi_{q}^{ \pm}(x)=\frac{1}{\sqrt{2}}\left(\begin{array}{c}
1 \\
v_{ \pm}
\end{array}\right) e^{ \pm i q_{x} x}
$$

where $u_{ \pm}= \pm \operatorname{sign}(E)$ and $v_{ \pm}=\frac{E-V(x)}{\hbar v_{F}\left( \pm q_{x}-i k_{y}\right)}$. Using these equations, one establishes the continuity conditions when the wave goes from the $k$-region to the $q$-region.

With the potential described in Fig. 1 at hand, we compute the transmission coefficient, defined as

$$
T_{G}(E)=\frac{\left|\psi_{k}^{ \pm}(0)\right|^{2}}{\left|\psi_{k}^{ \pm}(L t)\right|^{2}}
$$

using the transfer matrix formalism 30, 35, 36]. The transfer matrix can be obtained by imposing the continuity conditions of the wave functions in each region of the self-similar structure. From this we can extract the transmission coefficient for a given energy, see for instance [30, 35].

Since the construction of the multibarrier structure is based on the length-scaling of the previous generation barriers, and the addition of energy-scaled new barriers, we search for relations between transmission curves corresponding to different generations of the multibarrier structure. So, we fix the total length of the structure, denoted by $L t$ (in this case we use the value of $L t=3500 \AA$ ) and the height of the main barrier, denoted by $V_{0}$ (using the value of $V_{0}=1 \mathrm{eV}$ ). 
a) Generation comparison.

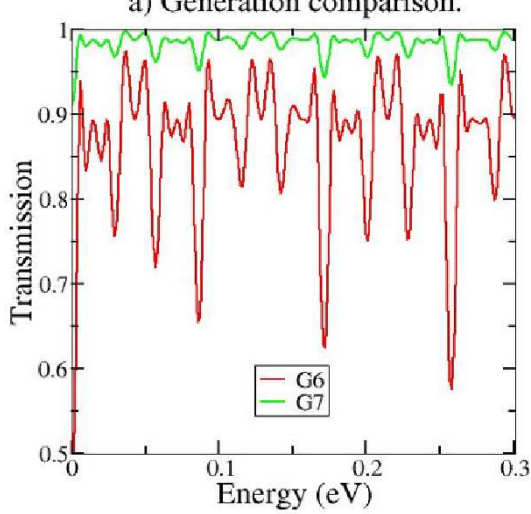

c) Comparison, Schrödinger case.

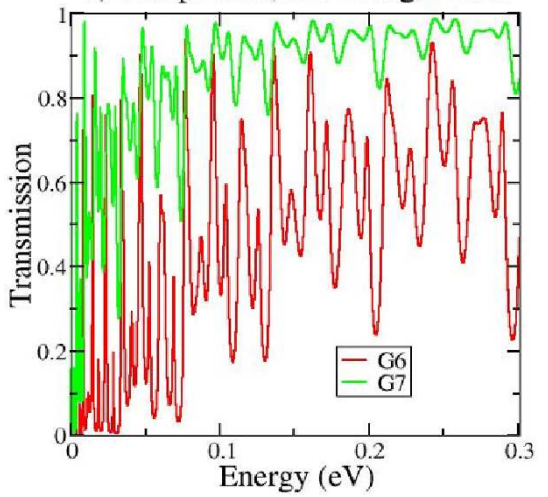

b) Scaling generation 7 .

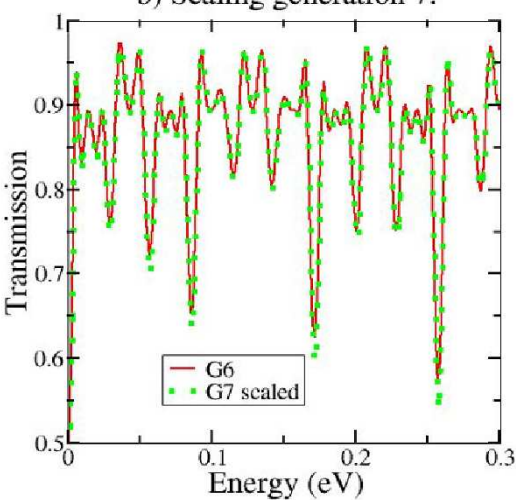

d) Scaling, Schrödinger case.

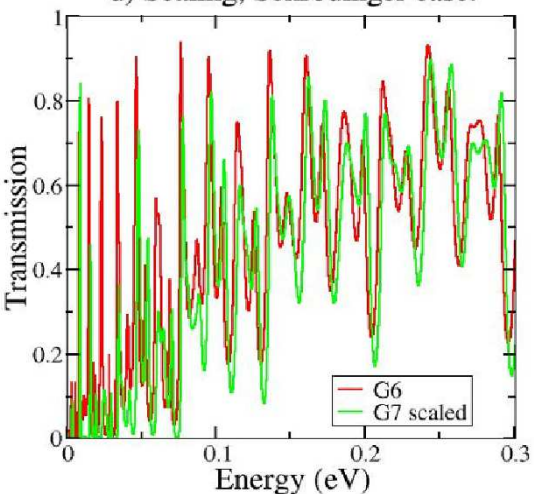

FIG. 2: Transmission coefficient scaling for different generations. a) Transmission as a function of energy for the sixth $(G=6)$ and seventh $(G=7)$ generation of multibarrier graphene using the parameters $V_{0}=1.0 \mathrm{eV}$ and $L t=3500 \AA$. b) Scaled transmission for $G=6$ and $G=7$. The scaling transformation is given by the power law Eq.

(5), which gives an excellent agreement of the curves. In c) and d), the transmission and scaled transmission respectively are presented for the same potential but for a typical semiconductor which follows the Schrödinger equation. No self-similar scaling is observed.

In figure 2 a we show the transmission curves corresponding to generations 6 and 7 of the potential, denoted by $T_{6}(E)$ and $T_{7}(E)$ respectively. As we can see, it is qualitatively clear that they are related by some kind of transformation. This transformation turns out to be $T_{6}(E)=\left[T_{7}(E)\right]^{9}$, where the subindex stands for the generation number, as seen in figure $2 \mathrm{~b}$. Thus, $T_{6}(E)$ are $T_{7}(E)$ scaled by a single exponent. As may be expected, other bigger generations also follow the same rule. More formaly

$$
T_{G}(E) \approx\left[T_{G+1}(E)\right]^{9}
$$

where $G \geq 6$ and $G$ stands for the generation number. This means that any two transmission curves that differ only in the generation of their correspondig potential, and are related by some power of the form $9^{m}$, where $m$ is the difference between the generations. This suggests that the transmission curves follows an scaling rule which is a signature of the self-similar phenomena. In fact, here we are in presence of the first true self-similar physical property of graphene. Morever, even for perfect fractals it is known that the physical properties usually follow a multifractal behavior [23, 37] but here only one single fractal exponent is obtained.

It is important to remark here that there are always finite size effects due to the break in the scaling of the potential, which affects the first generations. In this particular case, one cannot extend self-similarity beyond the length of the system. This effect is explained in the supplementary section where we present the transmittance from generation $G 1$ to $G 9$. For low generations, the transmittance is basically dominated by the biggest energy barrier. Between $G 5$ and $G 6$, there is a dramatic change in the behavior since now the system has transmittance for energies lower than the main barrier height. As generations increase, scaling appears. Generation 6 is the crossover since as shown in the supplementary section, geometrical finite size effects in the potential die out after it. Also, is clear that in real systems there is also another finite size effect at the bottom of the hierarchy, where the Dirac approach can not be 
made due to the rapid variation of the potential at atomic distances.

To highlight the difference with a typical Schrödinger semiconductor, in figure 2 panels c) and d), we present the transmission calculated for the same potential generations using $\mathrm{Al}_{x} \mathrm{Ga}_{1-x} \mathrm{As} / \mathrm{GaAs}$. In such system, the height of the quantum wells are controlled by the Aluminium concentration $(x)$.

So far, we have found a scaling between transmission curves that correspond to different generations of the potential, but it seems possible to extend the search for scaling features to other parameters. We have two options at hand, the energy of the starting barrier and the total length of the structure.
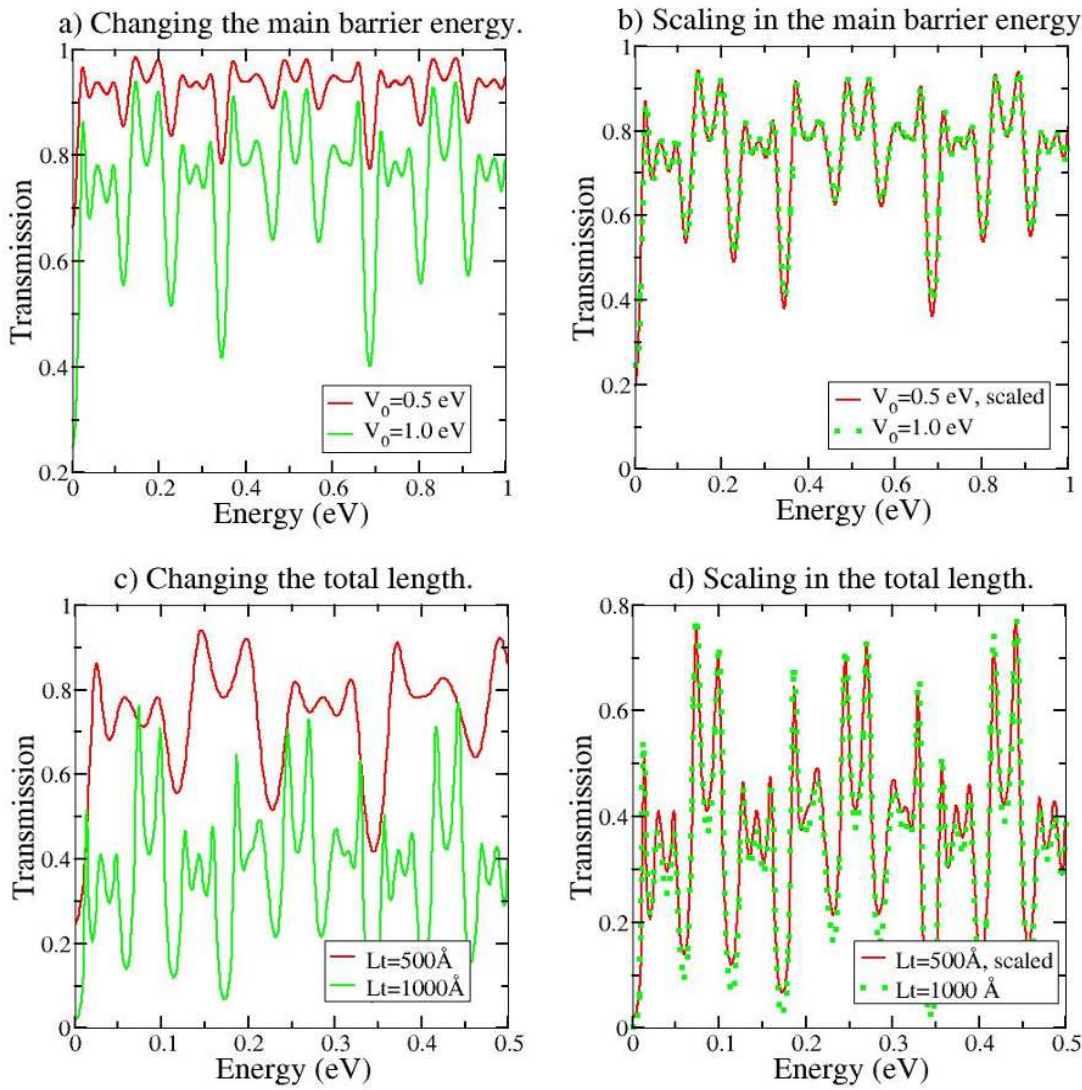

FIG. 3: Scaling between barrier energy. a) Comparison between transmission curves for different values of $V_{0}$ using generation 6 . This case corresponds to the transmission for two potentials with different heights of the main barrier, in this case $V_{0}=0.5 \mathrm{eV}$ and $V_{0}=1.0 \mathrm{eV}$. All other parameters are equal. b) Comparison between the scaled plots of a). The scaling consists in raising $V_{0}=0.5 \mathrm{eV}$ to the fourth power. Scaling between lengths. c) Comparison between the transmission curves for different lengths $(L t)$. In this case it is quite clear that the scaling cannot be just raising one curve to a certain power. d) Scaling of the curve with length $L t=500 \AA$. This scaling is made of two parts, raising the curve to the fourth power and multiplying its energy by two.

Let us explore now the first option, that is, we take a fixed generation of the potential, say generation 6 , and make the calculation for two starting energies. Lets take $V_{0}=0.5 \mathrm{eV}$ and $V_{0}=1.0 \mathrm{eV}$. In this case, the appearance of the curves seen in figure $3 \mathrm{a}$, resembles those corresponding to a change in the generation of the potential. In the case of the nonrelativistic transmission coefficient (e. g. GaAs), for the same geometric set up, one observes in general a shift of the first transmission resonance. Nevertheless, in this case the general behavior of the transmission curve is qualitatively the same. Due to the resemblance of these curves with the ones of changing generations, we try the same kind of transformation but with a different power. In this case, one has to raise the curve corresponding to $V_{0}=0.5 \mathrm{eV}$ to the fourth power to get the one corresponding to $V_{0}=1.0 \mathrm{eV}$, see figure 3 b. Thus $T_{1.0}(E) \approx T_{0.5}(E)^{4}$, where the subindex stands for the $V_{0}$ of the respective structure. In fact, this scaling behavior is more general, i. e., it applies for every multiple of two, resulting in the expression

$$
T_{\frac{1}{k} V_{0}}(E)^{k^{2}} \approx T_{V_{0}}(E) .
$$

Finally we compare curves corresponding to different lengths of the whole structure, denoted by $L t$. Although 
nothing suggests scaling in this variant at all, see figure 3r, our previous results lead us to a different conclusion. From the results for the case of changing $V_{0}$, we try to find a similar scaling law for $L t$, but in this case, the scaling in the energy gives the relation,

$$
T_{\frac{1}{\alpha} L t}\left(\frac{1}{\alpha} E\right)^{\alpha^{2}} \approx T_{L t}(E)
$$

With this scaling rule applied to the curve corresponding to $500 \AA$, a very good approximation is obtained, see figure 3].

Combining expressions (5), (6) and (7), it is possible to get a good approximation to almost every curve that results from the combination of parameters. To do this, all the parameters and the variable $E$ will be arguments of $T$,

$$
T\left(E, G, V_{0}, L t\right) \approx T\left(\alpha E, G-m, \frac{1}{k} V_{0}, \alpha L t\right)^{k^{2} / \alpha^{2} 9^{m}}
$$

for $G-m \geq 6$. All these approximations are quite useful to predict the transmission as function of the parameters, but it is necesary to test the correctness. So we calculate the root mean square of the difference between the target curve and the scaled one, we call it drms. For parameter $G$ (scaling between generations of the potential, see figure $2 \mathrm{~b}$ ) the $d r m s$ is $5.50 \times 10^{-5}$; for $V_{0}$ (scaling in the energy of the main barrier, see figure $3 \mathrm{~b}$ ) the $d r m s$ is $2.05 \times 10^{-4}$; at last for $L t$ (scaling in the total length of the multibarrier structure, see figure $3 \mathrm{~d}$ ) the $d r m s$ is $1.43 \times 10^{-3}$. From this it is quite clear that the better approximation is for the scaling between generations.

This lead us to examinate a wider range of energy and check our scaling-laws values (see supplementary material). An analysis of the results, shows that the fit is excellent for small energies but tends to decrease in quality as the energy increases, as one should expect in the limiting case of very high energies. Also, the error tends to grow for small transmittance energies. However, as seen in the supplementary material, if the error is computed for bigger generations, for example, for $G=7$ against scaled $G=8$, then the error decreases in a dramatic way, as expected for a good scaling relation. The drms for the Schroedinger case does not reduce as the generations grow, suggesting the absence of a fixed point.

The numerical results presented above suggests that this type of potential indeed shows scaling properties, although this scaling is not found in a single curve, but between curves corresponding to different parameters. This is a very interesting result because in fact the transmission coefficient is an intricate combination of the wave functions, and even more, for a multibarrier potential it is the result of the product of several transfer matrices. This suggests that the wave function have self-similar properties. That is the affirmation in the Gaggero-Pujals theorem [38] for nonrelativistic electrons. Thus, it seems plausible that an extension of that theorem could be established for relativistic equations. Another important possibility is to study the nonperpedicular incidence, since Klein tunneling is ruled out in this case [39].

In conclusion, the self-similar potential proposed here show various kinds of scaling properties in the transmission curves. This scaling properties gave the possibility to obtain, given a transmission curve and its set of parameters, an almost perfect approximation of the curve correspondig to certain transformation of the original parameters, see (8). It is also suggested that the scaling properties of the transmission curves are drastically affected by the mix of two geometric symmetries, for example, reflection and self-similarity, see [38, 40]. We believe that the proposed potential is ideal to study scaling properties in multibarrier systems, although our preliminary results considering electrostatic self-similar barriers in grafene indicate also the presence of a self-similar behavior.

Finally, we found that two ingredients are needed in order to obtain a pure monofractal scaling of the transmittance, a self-similar barrier and quantum relativistc equations, since the non-relativistic version does not display scaling. A possible heuristic explanation for such phenomena lies in the linear energy-momentum dispersion relation of the Dirac equation.

[1] Novoselov, K. S. et al. Electric Field Effect in Atomically Thin Carbon Films. Science, (306), 666-669 (2004).

[2] Novoselov, K.S. et al. Two-dimensional atomic crystals Proc. Natl. Acad. Sci. USA, 102 10451-10453 (2005).

[3] Balandin, A.A. et al. Superior thermal conductivity of single-layer graphene. Nano Lett., 8, 902-907 (2008).

[4] Geim, A. K., \& Novoselov, K. S. The rise of graphene. Nat. Mater. 6, 183-191 (2007).

[5] Novoselov, K. S. Nobel lecture: Graphene: Materials in the flatland. Rev. Mod. Phys., 83 837-849 (2011).

[6] Stone, D. A., Downing, C. A., \& Portnoi, M. E. Searching for Confined Modes in Graphene Channels: The Variable Phase Method Phys. Rev. B, 86075464 (2012).

[7] Naumis, G. G., Barrio, R. A., \& Wang, C. Effects of frustration and localization of states in the Penrose lattice. Phys. Rev. B, 50 9834-9842 (1994). 
[8] Naumis, G. G., Wang, C., \& Barrio, R. A. Frustration effects on the electronic density of states of a random binary alloy Phys. Rev. B, 65134203 (2002).

[9] Castro Neto, A. H., Guinea, F., Peres, N. M. R., Novoselov, K. S., \& Geim, A. K. The electronic properties of graphene. Rev. Mod. Phys., 81 109-162 (2009).

[10] Giovannetti, G., Khomyakov, P. A., Brocks, G., Kelly, P. J., \& van den Brink, J. Substrate-induced band gap in graphene on hexagonal boron nitride: Ab initio density functional calculations. Phys. Rev. B, 76073103 (2007).

[11] Corral, A. Long-term clustering, scaling, and universality in the temporal occurrence of earthquakes. Phys. Rev. Lett. 92 108501 (2004).

[12] Eloy, C. Leonardos Rule, Self-Similarity, and Wind-Induced Stresses in Trees Phys. Rev. Lett., 107, 258101 (2011).

[13] Harte, J., Kinzig, A., \& Green, J. Self-similarity in the distribution and abundance of species Science, 284 $334-336$ (1999).

[14] Song, C., Havlin, S., \& Makse, H. A. Origins of fractality in the growth of complex networks Nat. Phys., 2 275-281 (2006).

[15] Ding, H. X., Shen, Z. H., Ni, X. W., \& Zhu, X. F. Multi-splitting and self-similarity of band gap structures in quasi-periodic plates of Cantor series. Appl. Phys. Lett., 100, 083501 (2012).

[16] Miyamaru, F. et al. Emission of terahertz radiations from fractal antennas Appl. Phys. Lett., 95221111 (2009).

[17] Sun, M. et al. Transmission properties of dual-band cross-dipole fractal slit arrays for near- and mid-infrared wavelengths Phys. Rev. B, $\mathbf{7 4} 193404$ (2006).

[18] Maciá, E. The role of aperiodic order in science and technology. Rep. Prog. Phys., 69397 (2006).

[19] Hou, B., Xu, G., Wen, W., \& Wong, G. K. L. Diffraction by an optical fractal grating Appl. Phys. Lett. 85 6125 (2004).

[20] Estevez, J. O., Arriaga, J., Méndez-Blas, A., Robles-Cháirez, M. G., \& Contreras-Solorio, D. A. Experimental realization of the porous silicon optical multilayers based on the 1-s sequence J. Appl. Phys., 111013103 (2012).

[21] Lavrinenko, A. V., Zhukovsky, S. V., Sandomirski, K. S., \& Gaponenko, S. V. Propagation of classical waves in nonperiodic media: Scaling properties of an optical Cantor filter Phys. Rev. E, 65036621 (2002).

[22] Naumis, G.G., \& Aragon, J. L. Substitutional disorder in a Fibonacci chain: Resonant eigenstates and instability of the spectrum Phys. Rev. B, 54, 15079 (1996).

[23] Nava, R., Tagueña-Martínez, J., del Rio, J. A., \& Naumis, G. G. Perfect light transmission in Fibonacci arrays of dielectric multilayers. J. Phys.: Condens. Matter, 21, 155901 (2009).

[24] Cantor, Georg. Ueber unendliche, lineare punktmannichfaltigkeiten. Mathematische Annalen, 21, 545-591 (1883).

[25] Apostol, Tom M. Mathematical Analysis. [92] (Addison-Wesley Publishing Company), 1983.

[26] Novoselov, K. S., et al. Two-dimensional gas of massless Dirac fermions in graphene. Nature, 438 197-200 (2005).

[27] Zhang, Y., Tan, Y. W., Stormer, H. L., \& Kim, P. Experimental observation of the quantum Hall effect and Berry's phase in graphene. Nature, 438 201-204 (2005).

[28] Beenakker, C. W. J., Sepkhanov, R. A., Akhmerov, A. R. \& Tworzydlo, J. Quantum Goos-Hänchen Effect in Graphene Phys. Rev. Lett., 102146804 (2009).

[29] Nguyen, V. H., Bournel, A., \& Dollfus, P. Resonant tunneling structures based on epitaxial graphene on SiC Semicond. Sci. Technol., 26125012 (2011).

[30] Gomes, J. Viana, \& Peres, N M R. Tunneling of Dirac electrons through spatial regions of finite mass. J. Phys.: Condens. Matter, 20325221 (2008).

[31] Zhou, S. Y. et al. Substrate-induced bandgap opening in epitaxial graphene. Nature Mater., 6 770-775 (2007).

[32] Cheianov, V. V, Fal'ko, V., \& Altshuler, B. L. The Focusing of Electron Flow and a Veselago Lens in Graphene $p$-n Junctions Science, 3151252 (2007).

[33] Hartmann, R. R., Robinson, N. J., \& Portnoi, M. E. Smooth Electron Waveguides in Graphene Phys. Rev. B, 81245431 (2010).

[34] Hartmann, R. R., \& Portnoi, M. E. Quasi-exact Solution to the Dirac Equation for the Hyperbolic-secant Potential Phys. Rev. A, 89012101 (2014).

[35] Rodríguez-Vargas, I., Madrigal-Melchor, J., \& Oubram, O. Resonant tunneling through double barrier graphene systems: A comparative study of Klein and non-Klein tunneling structures. J. Appl. Phys., 112073711 (2012).

[36] Markos, P. and Soukoulis, C. M. Wave Propagation: From Electrons to Photonic Crystals and Left-Handed Materials. [3-10] (Princeton University Press), 2008.

[37] Naumis, Gerardo G. Minimal multifractality in the spectrum of a quasiperiodic Hamiltonian Phys. Lett. A, 365, 171-174 (2007).

[38] Gaggero-Sager, L. M., Pujals, E., Diaz-Guerrero, D. S., \& Escorcia-Garcia, J. [SelfSimilarity in Semiconductors: Electronic and Optical Properties]. Optoelectronics - Materials and Techniques. [Prof. P. Predeep (Ed.)] [435-458] (InTech) $2011 . \quad$ Available at http://www.intechopen.com/books/optoelectronics-materials-and-techniques/self-similarity-in-semiconductors-electronic-and-o

[39] Katsnelson, M. I., Novoselov, K. S., \& Geim, A. K. Chiral tunnelling and the Klein paradox in graphene. Nat. Phys., 2, 620-625 (2006).

[40] Diaz-Guerrero, D. S., Montoya, F., Gaggero-Sager, L.M., \& Perez-Alvarez, R. Transmittance and fractality in a Cantor-like multibarrier system. Progress In Electromagnetics Research Letters, 2, 149-155 (2008). 